\title{
Miranda
}

Revue pluridisciplinaire du monde anglophone /

Multidisciplinary peer-reviewed journal on the English-

speaking world

$5 \mid 2011$

South and Race / Staging Mobility in the United States

\section{Joan Thomas, Curiosity}

Laurence Talairach-Vielmas

\section{OpenEdition}

\section{Journals}

Édition électronique

URL : http://journals.openedition.org/miranda/2559

DOI : 10.4000/miranda.2559

ISSN : 2108-6559

Éditeur

Université Toulouse - Jean Jaurès

Référence électronique

Laurence Talairach-Vielmas, " Joan Thomas, Curiosity », Miranda [En ligne], 5 | 2011, mis en ligne le 29 novembre 2011, consulté le 16 février 2021. URL : http://journals.openedition.org/miranda/2559 ; DOI : https://doi.org/10.4000/miranda.2559

Ce document a été généré automatiquement le 16 février 2021.

\section{$\Theta \Theta \Theta \Theta$}

Miranda is licensed under a Creative Commons Attribution-NonCommercial-NoDerivatives 4.0 International License. 


\title{
Joan Thomas, Curiosity
}

\author{
Laurence Talairach-Vielmas
}

\section{RÉFÉRENCE}

Joan Thomas, Curiosity (Toronto : McClelland \& Stewart, 2010), 409 p, ISBN 978-07710-

8417-1

1 Pour ceux et celles qui aiment voyager dans le temps, voici un roman qui permettra aux amateurs d'histoire des sciences de remonter aux premières décennies du dixneuvième siècle, à un moment où les découvertes de fossiles de plus en plus étranges bouleversent les éminents scientifiques réunis à la Société Géologique de Londres. Le décor est campé dès les premières pages: nous nous trouvons en plein débats préévolutionnistes, dans l'Angleterre des années 1820. A Paris, la tension monte entre Georges Cuvier (1769-1832) et Jean-Baptiste Lamarck (1744-1829) et Etienne Geoffroy Saint-Hilaire (1772-1844) qui avancent tous deux l'idée d'une transmutation possible des espèces. En Angleterre, par contre, la Genèse sert encore de traité d'histoire naturelle. Le roman nous fait voyager de l'Angleterre à la France, contrastant le conservatisme poussiéreux anglais et une pensée métamorphosée par la révolution qui tente de s'émanciper des modèles théoriques du passé, et qui depuis Les Epoques de la nature (1778) de Georges Louis Leclerc, comte de Buffon, replace déjà l'homme sur une échelle temporelle plus vaste. Cependant, en Angleterre, l'école britannique ne peut dissocier la science de l'histoire sacrée. A des lieues de la vision sécularisée de l'univers issue des philosophes des lumières l'histoire de la terre et celle de l'homme ne font qu'un. Les plus célèbres paléontologues de l'époque, de William Buckland (1784-1856) à William Daniel Conybeare (1787-1857) ou Adam Sedgwick (1785-1873), ne lisent d'ailleurs les fossiles que comme les reflets d'un plan de Création. Tous très proches de l'église anglicane, ils lisent le monde naturel comme une chaîne des êtres que les découvertes de fossiles permettraient de dévoiler chaque jour un peu plus.

2 Pourtant, comme le roman le montre, les travaux de Georges Cuvier arrivent bien en Angleterre, en particulier après la traduction de Théorie de la Terre (1813) (Essay on the 
Theory of the Earth [1813]). Mais les paléontologues anglais se servent des théories du pionnier de l'anatomie comparée pour renforcer les principes de la théologie naturelle. Fixiste et catastrophiste, Cuvier pense que l'existence des fossiles est le résultat de grandes catastrophes naturelles, qui ont été suivies de nouveaux processus de création et son anatomie fonctionnelle devient, pour les naturalistes anglais enseignant à Oxford et Cambridge, la preuve scientifique d'un plan de Création. L'idée d'extinction des espèces sous les pressions environnementales avancée par Cuvier cadre mal avec celle de l'Arche de Noé, destiné à la préservation de chaque espèce. Mais les nouvelles découvertes géologiques bouleversent chaque jour un peu plus la théologie naturelle, et l'idée que des fossiles précèdent l'apparition de l'homme met à mal le jardin d'Eden et la notion même de péché originel: comment expliquer la violence dont les fossiles témoignent, tout particulièrement lorsque les paléontologues se penchent sur les coprolithes, fèces de dinosaures révélant leurs pratiques cannibales-un monde de souffrance et de violence que l'on ne peut rattacher à la Chute?

C'est donc au milieu de ce cercle de scientifiques que Joan Thomas nous transporte à chaque page, dans le monde de l'undergroundology, nous permettant de relire l'histoire des sciences en contexte, et redonnant à Mary Anning sa place centrale dans les découvertes de nouveaux fossiles dans la région de Lyme Regis. L'époque est retranscrite à merveille. On découvre ou retrouve une quête obsessionnelle pour les origines de l'homme, que le roman fasse état de l'exposition de la Vénus hottentote à Londres ou que les descriptions de fossiles inquiètent ceux qui cherchent à relire l'histoire de l'Arche de Noé à la lumière des monstrueuses créatures que l'on déterre ou extrait des falaises de Lyme Regis. Les questionnements foisonnent, les dragons volent, nagent ou rampent, les fossiles mis bout à bout proposant de drôles de créatures qui inquiètent les scientifiques qui ne savent plus vraiment où placer l'homme parmi les êtres sauvés lors du déluge.

4 Aucun ingrédient ne manque à l'appel. Les illustres scientifiques de l'époque sont tous ou presque présents, et l'on se délecte à la lecture de leurs traits de caractère. La lecture se fait jeu de piste pour le lecteur amateur d'histoire des sciences, qui redécouvre à chaque page l'époque que Thomas fait revivre du bout de sa plume. Mais le roman est aussi une histoire d'amour, proposant une explication à certaines rumeurs sur Mary Anning, et choisissant parmi ses amants potentiels Henry de la Beche. Ce dessinateur satirique, fiancé à Letitia Whyte, ne peut pendant quelques temps se résoudre à endosser son role d'époux dans le beau monde et oublier celle dont le regard franc sait scruter êtres et falaises à la recherche de la vérité. Agile et intelligente bien que peu lettrée, Mary Anning fascine les scientifiques de passage à Lyme Regis qui n'hésiteront pas à acheter les célèbres fossiles bientôt exposés à Londres, comme à s'approprier ses découvertes. L'histoire met le doigt non seulement sur les débats de l'époque, mais aussi sur les secrets enfouis, l'impensable-une histoire d'amour entre une prolétaire au visage hâlé et aux bottes crottées et un aristocrate. La romance permet à Thomas de nous révéler une Mary Anning visionnaire, qui aura su voir avant les scientifiques par trop conservateurs l'histoire de la terre inscrite à même les falaises de Lyme Regis, dans les couches qu'Henry de la Beche redessinera pour elle, cartographiant le paysage en suivant ses conseils et regardant enfin le monde avec ses yeux. Si la complicité intellectuelle entre Mary Anning et Henry de la Beche n'aura pas raison des conventions, l'histoire d'amour nous fait pénétrer un monde où les femmes n'ont pas voix au chapitre. Elles peuvent ruser, à l'instar de Leticia, qui se fera épouser par de la Beche portant l'enfant d'un autre, mais elles sont exclues du monde 
scientifique, interdites de lectures trop intellectuelles ou qui affichent des corps mis à nu par la science, et d'autant plus lorsqu'elles savent à peine lire et ne lisent l'heure qu'en observant la nature. On achète leurs talents sans leur laisser le droit de passer à la postérité, on les épouse de peur que des rivaux profitent de leurs qualités, comme William Buckland qui convole brusquement en justes noces avec Mary Moreland, alors illustratrice de Cuvier, pour faire un pied de nez au scientifique français. Pourtant, l'intelligence de celle que la science a aujourd'hui oubliée se lit à chaque page, et le lecteur ne manquera pas de sourire à la vue des boucles d'oreille que Mary offrira à en cadeau de mariage à la jeune promise-des coprolithes, excréments d'Ichthyosaures qui se balancent au bout de deux crochets de pêche.

Curiosity, un roman qui n'a de cesse de tisser des liens entre science et société, nous offre un voyage au cœur de l'histoire de la terre et de celle l'homme. Thomas nous propose aussi son interprétation de l'histoire, choisissant de la Beche comme l'amant inconnu, celui qui vendra son Duria Antiquior (1830) au profit des Anning et dont le discours à la mort de Mary en 1847 permettra à son nom d'entrer dans les annales de la Société Géologique de Londres. La recherche du fossile introuvable et de l'amour se mêlent pour le plus grand bonheur du lecteur qui suit avidement les mots qui claquent et l'ironie mordante de Thomas, la douceur de ses phrases comme son ton incisif et parfois caustique. Mais Curiosity est aussi une réflexion sur l'humain-un récit qui, en plaçant l'homme entre raison et sentiments, donne à la science un rôle central dans notre lecture du monde et de l'autre/Autre. Pour ceux qui ont été déçus par Remarkable Creatures de Tracy Chevalier et tous les autres, une lecture incontournable et un écrivain à découvrir ou retrouver sans attendre.

INDEX

Keywords : curiosity

Mots-clés : curiosité

\section{AUTEURS}

\section{LAURENCE TALAIRACH-VIELMAS}

Professeur

Université Toulouse 2 - Le Mirail

talairac@univ-tlse2.fr 